Case Report

\title{
Acute Panmyelosis with Myelofibrosis - A Rare Subtype of Acute Myeloid Leukemia
}

Tathagata Chatterjee ${ }^{2}$, Srishti Gupta ${ }^{1}$, Ajay Sharma ${ }^{1}$, Sanjeevan Sharma ${ }^{1}$ and Devika Gupta ${ }^{2}$.

${ }^{1}$ Department of Pathology and Molecular Medicine, Army Hospital (Research and Referral), Delhi Cantt - 110010 India

${ }^{2}$ Department of Immunohematology and Transfusion Medicine, AFMC, Pune - 411040 India

Correspondence to: Dr Srishti Gupta, Department of Pathology and Molecular Medicine Army Hospital (Research and Referral), Delhi Cantt - 110010. Tel: +91-9910760125. E-mail: srishtigpt10@gmail.com

Competing interests: The authors have declared that no competing interests exist.

Published: June 4, 2013

Received: March 28, 2013

Accepted: June 2, 2013

Citation: Mediterr J Hematol Infect Dis 2013, 5(1): e2013042, DOI: 10.4084/MJHID.2013.042

This article is available from: http://www.mjhid.org/article/view/11647

This is an Open Access article distributed under the terms of the Creative Commons Attribution License (http://creativecommons.org/licenses/by/2.0), which permits unrestricted use, distribution, and reproduction in any medium, provided the original work is properly cited.

Abstract. One case of acute panmyelosis with myelofibrosis (APMF) is here reported. A 45 year old male presented with abrupt onset of rapidly progressing low backache, weakness and pancytopenia. On examination there was no organomegaly. Peripheral blood examination revealed normocytic normochromic red blood cells with $10 \%$ circulating blasts. Flowcytometric examination of peripheral blood revealed blasts which were positive for CD34, HLA- DR and myeloid associated antigens (i.e. CD13 and CD33). Blasts were negative for anti MPO. Bone marrow aspirate resulted in a dry tap. Bone marrow biopsy revealed panmyeloid proliferation with scattered blasts which were CD34 positive on imunohistochemistry and negative for anti MPO. Reticulin stain showed grade III myelofibrosis (WHO). Differential diagnosis considered included AML-M7, MDS-RAEB II and AML with myelodysplasia. He was started on chemotherapy [idarubicin and cytarabine; 3+7 induction regimen followed by three cycles of HIDAC (High dose cytosine arabinoside)] after which patient was in complete morphological remission with markedly reduced bone marrow fibrosis. He is now being worked up for allogeneic stem cell transplantation. Patient is asymptomatic at eight months of diagnosis. In conclusion these patients should be managed aggressively with AML therapy and this case report reaffirms the fact that APMF is subtype of AML.

Introduction. Acute panmyelosis with myelofibrosis (APMF) is a rare form of acute myeloid leukemia and is characterized by acute panmyeloid proliferation with increased blasts and accompanying fibrosis of the bone marrow that does not meet the criteria for AML with myelodysplasia related changes. ${ }^{1}$

APMF is classified under acute myeloid leukemia not otherwise specified by WHO 2008. ${ }^{1}$ This entity is distinct and needs to be distinguished from acute megakaryoblastic leukemia (AML-M7), myelodysplastic syndrome - refractory anemia with excess blast II ( MDS-RAEB-II) with fibrosis, primary myelofibrosis (PMF) and AML with myelodysplasia related changes. ${ }^{2}$ The clinical course of this entity is rapidly progressive and fatal, therefore, it is essential to be aware of this entity and distinguish it from its mimickers. ${ }^{1,2}$ Though it may be extremely difficult to differentiate APMF from its mimickers in some cases, 
detailed clinical history and hematological work up can be helpful in such cases. Many consider, as evidenced by many published articles, that APMF is a variant of MDS. ${ }^{3,4,5,6}$ Since the outcome of these patients is poor therefore it is important to aggressively manage these patients with timely diagnosis as it can reduce morbidity and prolong life. In this case report other differential diagnosis considered are highlighted.

Case presentation. A 45 year old male presented with acute onset rapidly progressive low backache, fatigue and weakness for past one month. On examination he had no organomegaly, lymphadenopathy, petechiae or purpura. There was no significant past and family history. There was no history of any cytotoxic drug intake. Peripheral blood examination revealed pancytopenia. Total leukocyte count was $3000 / \mathrm{mm}^{3}$ with a platelet count of $40000 / \mathrm{mm}^{3}$ and hemoglobin level of $7 \mathrm{gm} / \mathrm{dl}$. Red blood cells were normocytic normochromic with minimal anisopoikilocytosis. Eight nucleated red blood cells were seen per hundred white blood cells. Occasional fully chromic tear drop cells were also noted. Differential leukocyte count comprised of blast $10 \%$, polymorphonuclear leukocyte $65 \%$, lymphocyte $23 \%$, eosinophil $02 \%$, basophil $0 \%$. Blasts morphologically were myeloblast with an occasional auer rod. However cytochemical MPO stain on peripheral blood smear was negative.

Flow cytometric analysis was done on peripheral blood using Beckman Coulter FC 500 flowcytometer (Beckman Coulter, Miami, FL, USA). Gating strategy was SSC vs CD45.An antigen was considered positive if greater than $20 \%$ of blasts gated showed positivity
Table 1. Flowcytometric result of peripheral blood sample, Gating strategy-SSC Vs CD45, Beckman Coulter FC 500, Events-50000.

\begin{tabular}{|l|c|c|}
\hline Antibody & $\begin{array}{c}\text { Result } \\
\text { (Percentage } \\
\text { positivity) }\end{array}$ & Intensity \\
\hline CD34 & $46 \%$ & Dim to Moderate \\
\hline CD13 & $55 \%$ & Dim to Moderate \\
\hline CD33 & $66.3 \%$ & Dim to Moderate \\
\hline CD45 & $78.9 \%$ & Moderate \\
\hline HLA-DR & $45.9 \%$ & Dim to moderate \\
\hline CD117 & $16.5 \%$ & Dim \\
\hline CD4 & $46 \%$ & Dim to moderate \\
\hline CD19 & $2.9 \%$ & Negative \\
\hline CD10 & $2.5 \%$ & Negative \\
\hline CD15 & $21.7 \%$ & Strong \\
\hline CD7 & $1.6 \%$ & Negative \\
\hline CD3 & $0.2 \%$ & Negative \\
\hline CD8 & $0.4 \%$ & Negative \\
\hline Anti cMPO & $5.5 \%$ & Negative \\
\hline CD14 & $1.4 \%$ & Negative \\
\hline CD11c & $9.7 \%$ & Negative \\
\hline cCD79a & $8.4 \%$ & Negative \\
\hline cCD3 & $0.2 \%$ & Negative \\
\hline
\end{tabular}

for a specific monoclonal antibody. Antibodies used were CD19, CD10, CD34, anti-MPO, HLA DR, CD11c, CD14, CD4, CD8, CD3, cCD79a, cCD3, CD117, CD15, CD13, CD33 and CD7. Antibodies against CD41, CD61, glycophorin A could not be used as they were not available at that time. $4.1 \%$ blasts were gated which were CD45 positive (moderate) and had low side scatter. $46 \%$ of gated blasts were positive
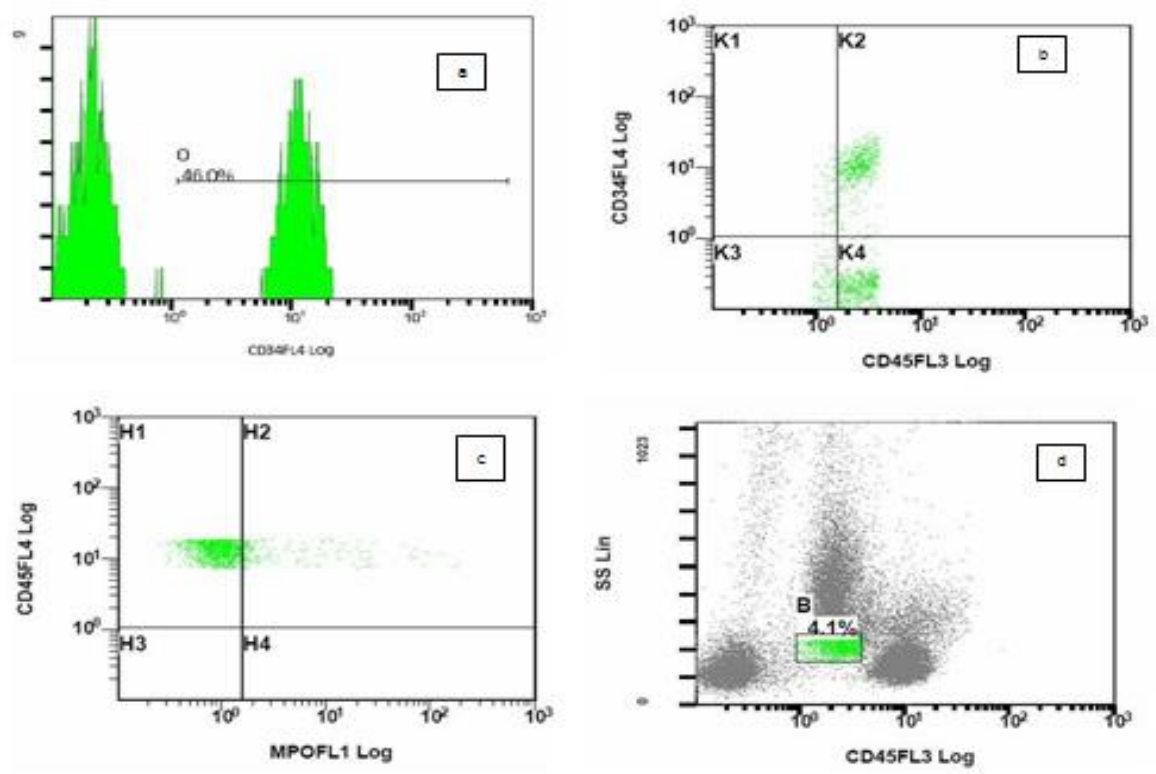

Figure 1. Figure 1 shows flowcytometric analysis on peripheral blood of CD34 antigen on blast population (a) shows histogram of CD34+blasts (B) shows scatter diagram of CD34 vs CD 45.Blasts are CD34 + and CD45 + (c) shows scatter diagram of CD 45 vs cMPO. Blasts are negative for cytoplasmic myeloperoxidase (d) Figure shows $4.1 \%$ blasts gated using CD45 vs SSC gating strategy 
for CD34 (Figure 1). Flow cytometry result is shown in table 1.

Bone marrow aspirate was unsuccessful but bone marrow biopsy (Figure 2) revealed hypercellular bone marrow with panmyeloid proliferation. Dyspoietic megakaryocytes, immature myeloid precursors and erythroid precursors were seen. Numerous small megakaryocytes were noted with hypolobated and nonlobated nuclei and dispersed chromatin. Blasts were seen dispersed as well as in clusters. However no atypical localization of immature precursors was seen. Reticulin stain revealed a characteristic WHO grade III myelofibrosis $^{7}$ (Figure 3).

Immunohistochemistry was done on paraffin embedded sections using automated immunostainer (DAKO, CA, USA) based on standard streptavidin biotin-peroxidase complex technique. Antibodies used were antiCD61, anti glycophorin A, antiCD34 and anti MPO. Blasts were positive for CD34 (Figure 4) and negative for CD61, anti MPO and glycophorin A.

JAK2 mutation analysis was carried out and

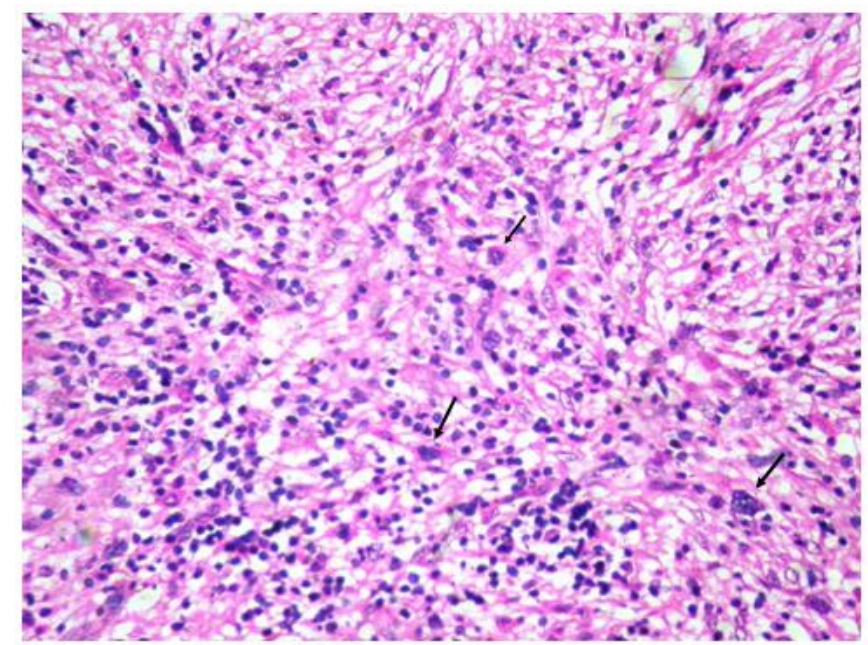

Figure 2. Figure 2 shows panmyeloid proliferation with dyspoietic unilobated megakaryocytes(arrow). (Hematoxylin and eosin $\mathrm{x} 40 \mathrm{x}$ ).

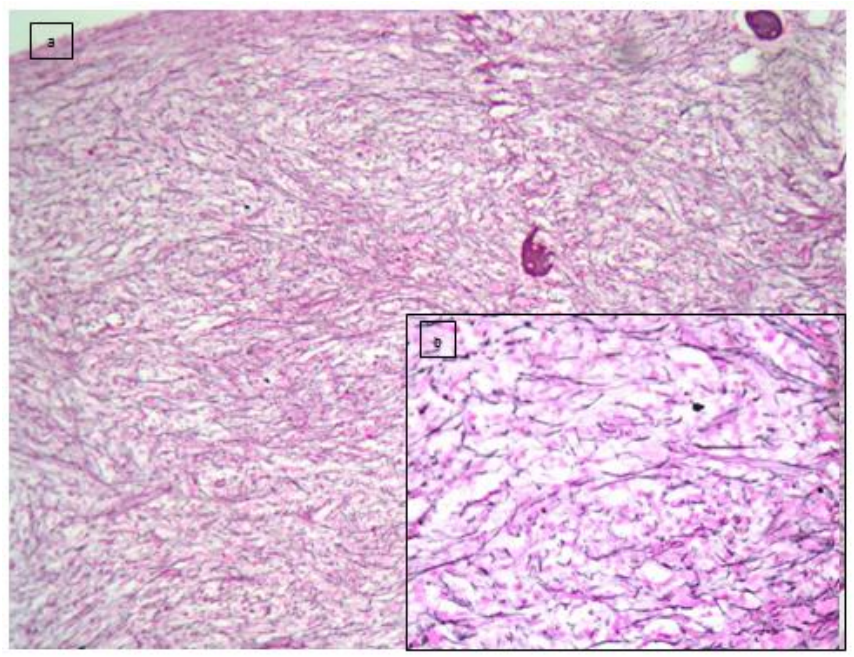

Figure 3. a \& b (inset) shows increased WHO grade III fibrosis on reticulin stain(Gomori silver impregnation technique(a-10x)(b-40x).

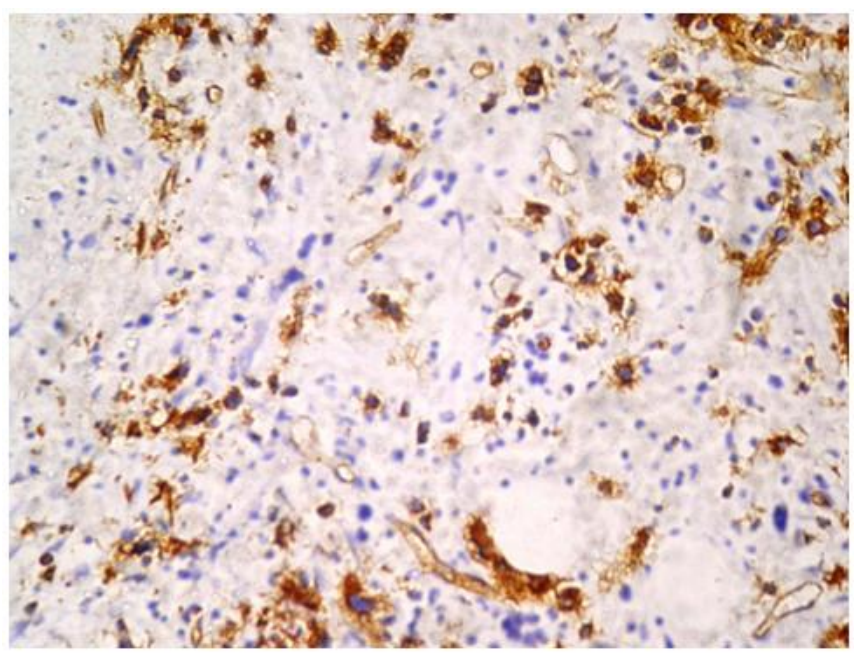

Figure 4. Figure 4 shows CD 34 positive blasts with a working internal control (arrow-endothelium) (10x) (DAKO automated immunostainer- streptavidin biotin - peroxidase based technique).

revealed a wild type mutant. Conventional cytogenetic analysis was carried out to rule out any abnormal cytogenetics. However no abnormality was detected.

The patient was treated as a case of acute myeloid leukemia with a $3+7$ induction regimen of idarubicin $\left(12 \mathrm{mg} / \mathrm{m}^{2}\right)$ and cytarabine $\left(100 \mathrm{mg} / \mathrm{m}^{2}\right)$. This was followed by three consolidation cycles of HIDAC (High dose intermittent ARA-C; each cycle comprising of $4.5 \mathrm{~g} \mathrm{BD}$ for three days) which led to marked improvement in symptoms. Bone marrow biopsy done for remission status after 45 days of starting HIDAC regimen revealed a dramatic reduction in fibrosis to grade I. Blasts were reduced to less than 5\%. Patient is being planned for autologous hematopoietic stem cell transplant due to non-availability of HLA-matched donor.

Result. The outcome of such patients is usually poor with a rapidly fatal course. Above patient has responded to chemotherapy as evidenced by symptomatic improvement, decrease in blast percentage to less than $5 \%$ and reversal of myelofibrosis from grade III to grade I. Patient is asymptomatic at eight months of diagnosis and has not had any episode of relapse.

Discussion. Differential diagnosis entertained in this case included AML-M7, MDS-RAEB-2 with fibrosis, PMF and AML with myelodysplasia related changes. In AML-M7 blasts are more than $20 \%$ of all nucleated cells in peripheral blood and/or bone marrow and atleast $50 \%$ are megakaryoblasts (CD41/CD61 positive). ${ }^{1}$ This was not so in our case. Additionally blasts in APMF are always positive for CD34 and in AML M7 blasts are positive for CD34 only in $60 \%$ of the cases. ${ }^{2}$

It was difficult to differentiate APMF from MDS- 
RAEB II with fibrosis and this difficulty has been evidenced by many published manuscripts. ${ }^{2,5,8}$ MDSRAEB II with fibrosis was negated based on abrupt clinical presentation and immunohistochemistry result as blasts in our case were anti MPO negative. Also numerous small megakaryocytes with nonlobated and hypolobated nuclei with dispersed chromatin pointed towards a diagnosis of APMF. Lastly, the striking degree of myelofibrosis was also in favour of APMF. ${ }^{2}$

PMF was ruled out as there was no intrasinusoidal hematopoiesis. Also, characteristic tight cellular clusters of large megakaryocytes with clumped, hyperchromatic nuclei (cloud or balloon shape) were absent. ${ }^{9}$ AML with myelodysplasia related changes was ruled out as blasts were less than $20 \% .^{10}$

In view of abrupt onset of rapidly progressive symptoms in our case with panmyelosis a diagnosis of APMF was given.

APMF is a rare and aggressive form of AML and arises denovo. ${ }^{1}$ Many believe it responds poorly to chemotherapy and mean survival is 9 months. ${ }^{1}$ In our case aggressive management with AML chemotherapy

\section{References:}

1. Arber DA, Brunning RD, Orazi A, Porwit A, Peterson L, Thiele $\mathrm{J}$ et al. Acute myeloid leukemia not otherwise specified In: Swerdlow SH, Campo E, Harris NL, Jaffe ES, Pileri S A, Stein H, Thiele J, Vardiman JW (eds). World Health Organization Classification of Tumors, Tumors of Haematopoietic and Lymphoid Tissues. IARC Press: Lyon, 2008.

2. Orazi A, Malley DPO, Jiang J, Vance G H, Thomas $J$ et al. Acute panmyelosis with myelofibrosis:an entity distinct from acute megakaryoblastic leukemia. Modern Pathology (2005)18,603-614. http://dx.doi.org/10.1038/modpathol.3800348 PMid: 15578075

3. Sultan C, Sigaux F, Imbert M et al. Acute myelodysplasia with myelofibrosis; a report of eight cases. Br J Haematol 1981; 49:11-16. http://dx.doi.org/10.1111/j.1365-2141.1981.tb07191.x PMid:7272222

4. Allen EF, Lunde JH, McNally R, et al. A case of acute myelofibrosis with complex karyotypic changes: a type of myelodysplastic syndrome. Cancer Gene Cytogene 1996; 90: $24-$ 28. http://dx.doi.org/10.1016/0165-4608(96)00037-4

5. Thiele J, Kvasnika HM, Schmitt-Graeff A. Acute panmyelosis with myelofibrosis. Leuk Lymphoma 2004; 45: 681-687. http://dx.doi.org/10.1080/10428190310001625692 PMid:15160939

6. Thiele J, Kvasnika HM, Zerhusen G, Vardiman J, Diehl V, Luebbert M, Graeff AS. Acute panmyelosis with myelofibrosis: a clinicopathological study in 46 patients including gradually improved pancytopenia and reduced bone marrow fibrosis. This case highlights the importance of identifying and differentiating APMF from other entities like AML-M7 and RAEB-2 so that aggressive treatment can be instituted. This case also confirms that APMF is a type of AML unlike many who believed it to be a type of MDS., ${ }^{3,4,5,6}$

To conclude, in a patient with an abrupt onset of fever, bone pain and pancytopenia a diagnosis of APMF should be kept in mind. Such patients are usually adults with no organomegaly. The bone marrow (BM) tap is usually dry due to extensive fibrosis and BM biopsy features are characteristic. Panmyelosis with poorly differentiated blasts and scattered hypolobated and nonlobated megakaryocytes are noted. Immunophenotypic analysis usually shows blasts to be CD34, CD13, CD33 and CD 117 positive and negative for megakaryocytic lineage and myeloperoxidase. Cytogenetic analysis helps to differentiate APMF from its mimickers specially MDS RAEB2 and acute megakaryoblastic leukemia. ${ }^{1}$

histochemistry of bone marrow biopsies and follow up. Ann Hematol (2004) 83:513-521 http://dx.doi.org/10.1007/s00277004-0881-8 PMid:15173958

7. Thiele J, Kvasnika HM, Tefferi A, Barosi et al. Primary myelofibrosis. In: Swerdlow SH, Campo E, Harris NL, Jaffe ES, Pileri SA, Stein H, Thiele J, Vardiman JW (eds).World Health Organization Classification of Tumors, Tumors of Haematopoietic and Lymphoid Tissues. IARC Press: Lyon, France, 2008, p 46.

8. Steensma DP, Hanson CA, Letendre L, et al. Myelodysplasia with fibrosis: a distinct entity? Leuk Res 2001;25:829-838. http://dx.doi.org/10.1016/S0145-2126(01)00055-8

9. Thiele J, Kvasnika HM, Tefferi A, Barosi et al. Primary myelofibrosis. In: Swerdlow SH, Campo E, Harris NL, Jaffe ES, Pileri SA, Stein H, Thiele J, Vardiman JW (eds). World Health Organization Classification of Tumors, Tumors of Haematopoietic and Lymphoid Tissues. IARC Press: Lyon, France, 2008, p 45.

10. Arber DA, Brunning RD, Orazi A, Bain BJ, Porwit A, Vardiman $\mathrm{J} \mathrm{W}$ et al. Acute myeloid leukemia with myelodysplasia - related changes. In: Swerdlow SH, Campo E, Harris NL, Jaffe ES, Pileri SA, Stein H, Thiele J, Vardiman JW (eds). World Health Organization Classification of Tumors, Tumors of Haematopoietic and Lymphoid Tissues. IARC Press: Lyon, France, 2008, p124. 